\title{
Modulation Scheme Classification in Cognitive Radio Networks Using the Long Short Term Memory (LSTM) of Deep Learning
}

\author{
Isaac A. Oyedeji, Olumide O. Ajayi, and Sunday T. Aladesae
}

\begin{abstract}
Cognitive Radio (CR) system has been adopted for efficient utilization of radio frequency spectrum. The classification of signal modulation schemes is one of the main characteristics of the CR for appropriate demodulation of sensed signals. However, conventional Modulation Classification (MC) techniques require extensive extraction of signal features, which is not often guaranteed. Thus, Deep Learning (DL) has been seen as a promising solution to this drawback in MC. This paper proposes a DL-based MC technique using the Long Short-Term Memory (LSTM) network architecture. The proposed LSTM model was trained on M-ary Phase Shift Keying (MPSK) and M-ary Quadrature Amplitude Modulation (MQAM) signal types. The LSTM directly learns the features of a given modulation scheme of a signal sample during training. The signal samples were generated via computer simulation for Signal-to-Noise Ratios (SNRs) from -10 dB to $20 \mathrm{~dB}$ with an interval of $5 \mathrm{~dB}$ over flat fading channel and Additive white Gaussian noise (AWGN). Simulation results show that the proposed LSTM model achieves an average classification accuracy of $95 \%$ at SNRs above $0 \mathrm{~dB}$.
\end{abstract}

Keywords - Cognitive Radio (CR), Deep Learning (DL), Long Short-Term Memory (LSTM), Modulation Classification (MC).

\section{INTRODUCTION}

The continued global increase in the number of connecting devices for Device-to-Device (D2D) and Machine-toMachine (M2M) wireless communications such as the Internet of Things (IoT) [1], [2] has imposed a heavy demand for radio frequency spectrum. Consequently, the traditional spectrum access policy in which fixed frequency bands are assigned exclusively to licensed Primary Users (PUs) is no longer sustainable. This is because most of the frequency spectrum allocated to PUs are often underutilized; thereby creating the phenomenon called white spaces (or spectrum holes) [3].

In order to solve the problem of white spaces, the Cognitive Radio (CR) technology has been adopted [4]. The CR technology permits unlicensed Secondary Users (SUs) to make use of idle channels (i.e. white spaces) of PUs without causing interference to the PUs. Consequently, an SU that is willing to use an idle channel must apply a robust Spectrum Sensing (SS) technique to accurately detect the presence or absence of the PU in the channel so as to make the appropriate

Submitted on February 04, 2022.

Published on March 04, 2022.

I. A. Oyedeji, Ogun State Institute of Technology, Nigeria.

(e-mail: ayooyee@gmail.com)

O. O. Ajayi, Adeleke University, Nigeria. decision [5]. Some other functions that can be performed by a CR (i.e. an SU) are Adaptive Coded Modulation (ACM) and Modulation Classification (MC) [3], [6]

A CR is expected to be able to correctly recognize or classify the type of modulation scheme of the received signal so as to apply the appropriate demodulation process. However, MC by the CR is a challenging task if there is no prior knowledge of the signal characteristics like modulation scheme, coding, symbol rate and carrier frequency [3], [7]. Conventional MC requires signal feature extraction either at waveform level or symbol level; and the signal features can be categorized into temporal, spectral and vector-space [8], [9]. The drawbacks of feature-based MC techniques include high computational cost or high sensitivity to noise and distortion [3], [8], [10].

Recent developments in MC tend toward Machine Learning (ML) and Deep Learning (DL) techniques. In the ML techniques, signal features' extraction stage must precede the training of the classifier such as Artificial Neural Network (ANN), Support Vector Machine (SVM) and K-Nearest Neighbour (KNN) [11] - [14]. Consequently, the achievable classification accuracy depends on the quality of extracted signal features and the method of extraction, which are not usually guaranteed [15]. On the other hand, the DL techniques such Convolutional Neural Network (CNN) and Long Short-Term Memory (LSTM) do not require a separate feature extraction stage because the classifier learns to classify data directly [16], [17].

This paper investigates the performance of the LSTM classifier for recognizing the modulation scheme of a received signal by a CR. The LSTM classifier which is a DLbased technique is employed to address the problem of signal feature extraction during the MC process.

\section{RELATED WORK}

The DL approach for MC in Cognitive Radio Networks (CRNs) is gaining attention in recent times. The CNN model was proposed by Zhou et al. [16] for achieving robust MC at low Signal-to-Noise Ratios (SNRs). Constellation diagrams of modulation schemes were used to train the CNN model in [18]. A generalization strategy was used to enhance the standard CNN for MC in [15]. Zheng et al. [19] compared three fussion methods for handling variation in the length of the signal passed as input to the CNN classifier.

(e-mail: ajayi.olumide@ ${ }^{@}$ adelekeuniversity.edu.ng)

S. T. Aladesae, CITM Yaba College of Technology, Nigeria.

(e-mail: greatsae2@yahoo.com) 
In [20], a CNN classifier was trained with reduced signal dimensions and was shown to outperform some ML-based models. Lin et al. [21] proposed a combination of gated recurrent unit and $\mathrm{CNN}$ to detect temporal features. The superiority of the CNN-based MC over the classical models has also been revealed in [22].

Majority of the DL-based MC techniques proposed in the literature adopted the $\mathrm{CNN}$ model whereas little attention is given to the use of the LSTM model [23]. Thus, the application of the LSTM model for MC in CRN is the focus of this paper.

\section{SYSTEM MODEL}

\section{A. Cognitive Radio Link Model}

A typical communication link in a CRN with MC is shown in Fig. 1. The transmitter (e.g., a PU) modulates the information bits using either the M-ary Phase Shift Keying (MPSK) or M-ary Quadrature Amplitude Modulation (MQAM) digital modulation type [7]. The modulated signal is then transmitted over wireless fading channel. Additive white Gaussian noise (AWGN) is added to the faded received signal at the SU's receiver. The receiver has no prior knowledge of the modulation scheme used by the transmitter. Thus, the receiver applies an MC technique (which is LSTMbased in this study) to recognize the modulation scheme of the received baseband signal. Then the knowledge of the modulation scheme is used to demodulate the received signal so as to obtain the information.

Binary PSK (BPSK), Quadrature PSK (QPSK), 8PSK, 16QAM and 64QAM were selected for this study based on the IEEE 802.11a standard [9]. The signal received by a CR can be expressed as:

$$
y(t)=h(t) x(t)+z(t)
$$

where $x(t)$ is the transmit signal, $h(t)$ is the fading channel response and $z(t)$ is the AWGN which is zero-mean Gaussian random variables with unknown variance and $t$ is the time instant. The fading channel response $h(t)$ is assumed to be frequency non-selective fading, which is given by:

$$
h(t)=\alpha(t) e^{j \varphi(t)}
$$

where $\alpha(t)$ is a random variable taking from Rayleigh distribution, and $\varphi(t)$ is the phase [1]. [22]:

The MPSK modulated transmit signal can be expressed as

$$
x_{M P S K}(t)=\sum_{i} g\left(t-i T_{s}\right) \cos \left(2 \pi f_{c} t+(2 m+1) \frac{\pi}{M}\right)
$$

where $m \in\{0,1, \ldots, M-1\} . T_{s}$ is the symbol period, $g(\cdot)$ is the pulse and $M$ is the modulation level.

A transmit signal modulated by MQAM can be expressed as:

$$
x_{M Q A M}(t)=\sum_{i} g\left(t-i T_{s}\right) \sqrt{A_{i}^{2}+B_{i}^{2}} \cos \left(2 \pi f_{c} t+\varphi_{i}\right)
$$

where $\varphi_{i}=\tan ^{-1}\left(B_{i} / A_{i}\right), A_{i}$ and $B_{i}$ are functions of the constellation distance [22] .

It is evident in (3) and (4) that the modulation type of a received signal can be deduced from the signal characteristics or pattern. Thus, a pattern recognition system like the proposed LSTM model can be used to recognize the modulation scheme of a signal.

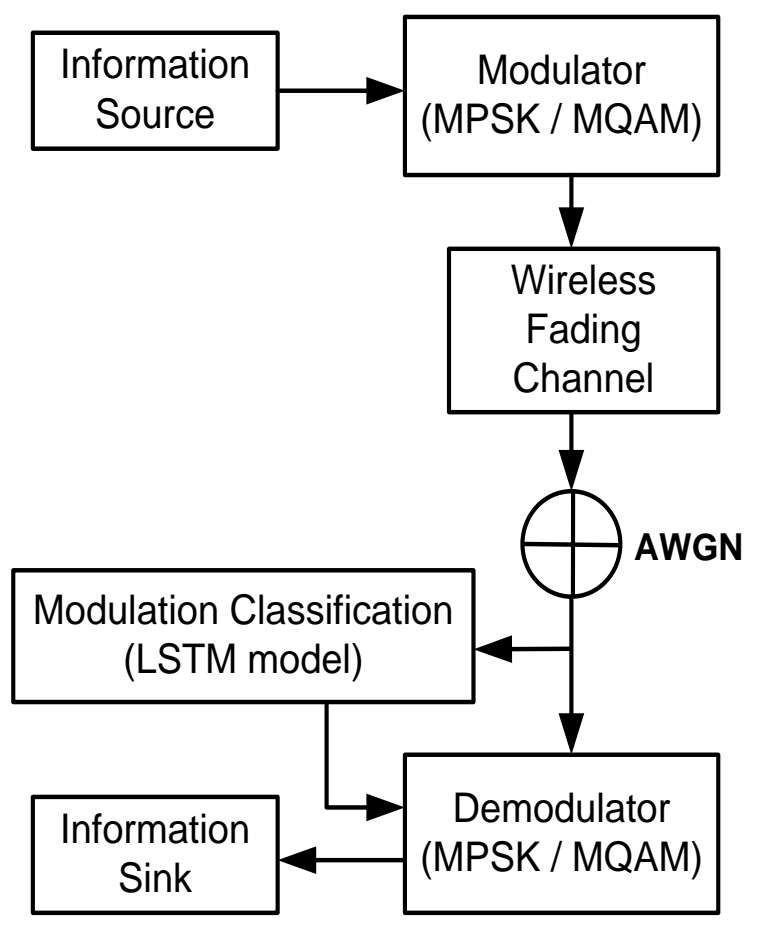

Fig. 1. Cognitive radio system with modulation classification.

\section{A. Proposed LSTM Model}

An LSTM network works by connecting the current state of information to the previous state in a time series [24]. The proposed LSTM model directly learns the features of a given modulation scheme of a known signal, and thereafter uses the knowledge to recognize the modulation scheme if present in an unknown signal. The architecture for the LSTM model with a single in the memory block is shown in Fig. 2. The hidden layer in the LSTM network is a recurrent layer that feeds the input units, input gate, forget gate and output gate from the output units of the cell. In practice, the LSTM network is made up of more than one memory block; and each block consists of one or more memory cells.

At a given iteration $t$, the LSTM maps input signal's sequence, $x(t)$, to the target output sequence, $y(t)$, using the network of unit activations; and all units are updated. The parameter $\mathrm{c}(\mathrm{t})$ is the cell activation vector at iteration $t, \mathrm{~W}_{\text {out }}$ is the matrix of weights from the output gate to the output, $\mathrm{W}_{\mathrm{f}}$ is the matrix of weights from the forget gate to the cell, $\mathrm{W}_{\text {in }}$ is the matrix of weights from the input gate to the input, whereas $\mathrm{h}$ and $\mathrm{g}$ denote the cell output and cell input activation functions, respectively.

The algorithm for the proposed LSTM model is summarized in Fig. 3. 


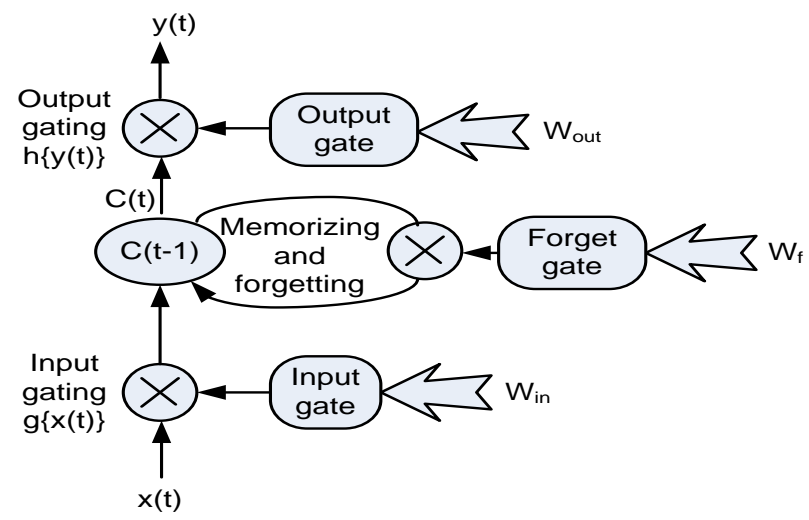

Fig. 2. Architecture for the LSTM model with a single cell.

\begin{tabular}{l}
\hline Algorithm: Proposed LSTM-based MC Technique \\
\hline Input: LSTM and CR communication parameters (information length, \\
SNR, carrier frequency, Signal samples size per modulation scheme, N, \\
etc.) \\
Output: Trained LSTM model for MC \\
BEGIN \\
1: Initialize empty sets for five datasets: BPSK, QPSK, 8PSK, 16QAM \\
and 64QAM modulation schemes \\
2: For Each modulation scheme \\
3: For $\mathrm{s}=1$ To N Do \\
4: Generate baseband transmit signal for each modulation scheme \\
5: Generate the Rayleigh fading channel and AWGN \\
6: Create the received signals using equation (1) \\
7: Compute the magnitude of the complex symbols \\
8: Update the datasets with the generated signal and target label \\
9: End For \\
10: End For \\
11: Merge the five datasets and randomly partition the resultant dataset \\
into training set and validation set \\
12: Create the LSTM model \\
13: Train the LSTM model on the training set \\
END
\end{tabular}

Fig. 3. Training of the proposed LSTM model for modulation classification.

\section{B. Training and Testing Methods}

For each of the modulation schemes \{BPSK, QPSK, 8PSK, 16QAM and 64QAM , information data consisting of 1000 bits was randomly generated and modulated. Then, the modulated waveform was mixed with the randomly generated fading process with the addition of AWGN process to obtain the received signal. This procedure was repeated to generate the signal samples under seven different SNRs: $-10 \mathrm{~dB},-5 \mathrm{~dB}$, $0 \mathrm{~dB}, 5 \mathrm{~dB}, 10 \mathrm{~dB}, 15 \mathrm{~dB}$ and $20 \mathrm{~dB}$. As contained in Table I, a combination of generated samples of three SNRs (i.e., $10 \mathrm{~dB}, 5 \mathrm{~dB}, 20 \mathrm{~dB}$ ) were used to train the LSTM model.

\begin{tabular}{ccc}
\multicolumn{3}{c}{ TABLE I: TRAINING DATASET SPECIFICATION } \\
\hline \hline $\begin{array}{c}\text { SNR } \\
(\mathrm{dB})\end{array}$ & Signal samples size per modulation scheme & Total \\
\hline-10 & BPSK (2000), QPSK (2000), 8PSK (2000), & \multirow{2}{*}{10000} \\
& 16QAM (2000), 64QAM (2000) \\
5 & BPSK (2000), QPSK (2000), 8PSK (2000), & 10000 \\
& 16QAM (2000), 64QAM (2000) \\
20 & BPSK (2000), QPSK (2000), 8PSK (2000), & 10000 \\
& 16QAM (2000), 64QAM (2000) \\
\hline
\end{tabular}

The training dataset was partitioned randomly in equal proportion into $80 \%$ for training and $20 \%$ for validation. The initial learning rate for the LSTM model was set to 0.01 . The adaptive moment estimation (adam) solver with batch size of 150 was adopted, and the maximum epoch set to 100 . The testing dataset was then passed as input to the trained LSTM model to classify the modulation scheme of each test signal sample. Summary of the testing dataset is contained in Table II.

\begin{tabular}{|c|c|c|}
\hline $\begin{array}{l}\text { SNR } \\
(\mathrm{dB})\end{array}$ & $\begin{array}{c}\text { Signal samples size per modulation } \\
\text { scheme }\end{array}$ & Total \\
\hline-10 & $\begin{array}{c}\text { BPSK (500), QPSK (500), 8PSK (500), } \\
\text { 16QAM (500), 64QAM (500) }\end{array}$ & 2500 \\
\hline-5 & $\begin{array}{c}\text { BPSK (500), QPSK (500), 8PSK (500), } \\
\text { 16QAM (500), 64QAM (500) }\end{array}$ & 2500 \\
\hline 0 & $\begin{array}{c}\text { BPSK (500), QPSK (500), 8PSK (500), } \\
\text { 16QAM (500), 64QAM }(500)\end{array}$ & 2500 \\
\hline 5 & $\begin{array}{l}\text { BPSK (500), QPSK (500), 8PSK (500), } \\
\text { 16QAM (500), 64QAM }(500)\end{array}$ & 2500 \\
\hline 10 & $\begin{array}{c}\text { BPSK (500), QPSK (500), 8PSK (500), } \\
\text { 16QAM (500), 64QAM }(500)\end{array}$ & 2500 \\
\hline 15 & $\begin{array}{c}\text { BPSK (500), QPSK (500), 8PSK (500), } \\
\text { 16QAM (500), 64QAM (500) }\end{array}$ & 2500 \\
\hline 20 & $\begin{array}{c}\text { BPSK (500), QPSK (500), 8PSK (500), } \\
\text { 16QAM (500), 64QAM (500) }\end{array}$ & 2500 \\
\hline
\end{tabular}

\section{RESUltS AND EVALUATION}

The system model was simulated with Matlab R2018a software package, and the performance of the proposed LSTM model was evaluated using classification accuracy. The graph of training accuracy and loss during training of the LSTM model for MC are shown in Fig. 4. Training accuracy of $99.96 \%$ was attained at $100^{\text {th }}$ epochs. Moreover, the loss of training could no longer be reduced after $85^{\text {th }}$ epoch.

The confusion matrix showing the classification performance of the trained LSTM model for MPSK modulation type (i.e., BPSK, QPSK and 8PSK) is presented in Fig. 5. The results reveal that the achieved overall classification accuracy is 93\%. Similarly, the overall classification accuracy of $77.6 \%$ was obtained for the MQAM type (i.e., 16QAM and 64QAM) as shown in the confusion matrix of Fig. 6 . The results clearly indicate that the LSTM model was able to recognize 8PSK better than both the BPSK and QPSK. Furthermore, the LSTM model could identify 64QAM better than 16QAM.

The performance of the proposed LTSM model for classifying the MSPK modulation type under SNRs from $-10 \mathrm{~dB}$ to $20 \mathrm{~dB}$ is presented in Fig. 7. The classification accuracy averaged over the range of SNRs for BPSK, QPSK and 8 PSK are $79.33 \%, 79.30 \%$ and $87.98 \%$, respectively. In addition, the proposed LSTM model achieves an average of $94.71 \%$ classification accuracy with SNR of $5 \mathrm{~dB}$ and above, whereas an average of $65.49 \%$ classification accuracy is obtained by the model in the low SNR region (i.e., $0 \mathrm{~dB}$ and below). 


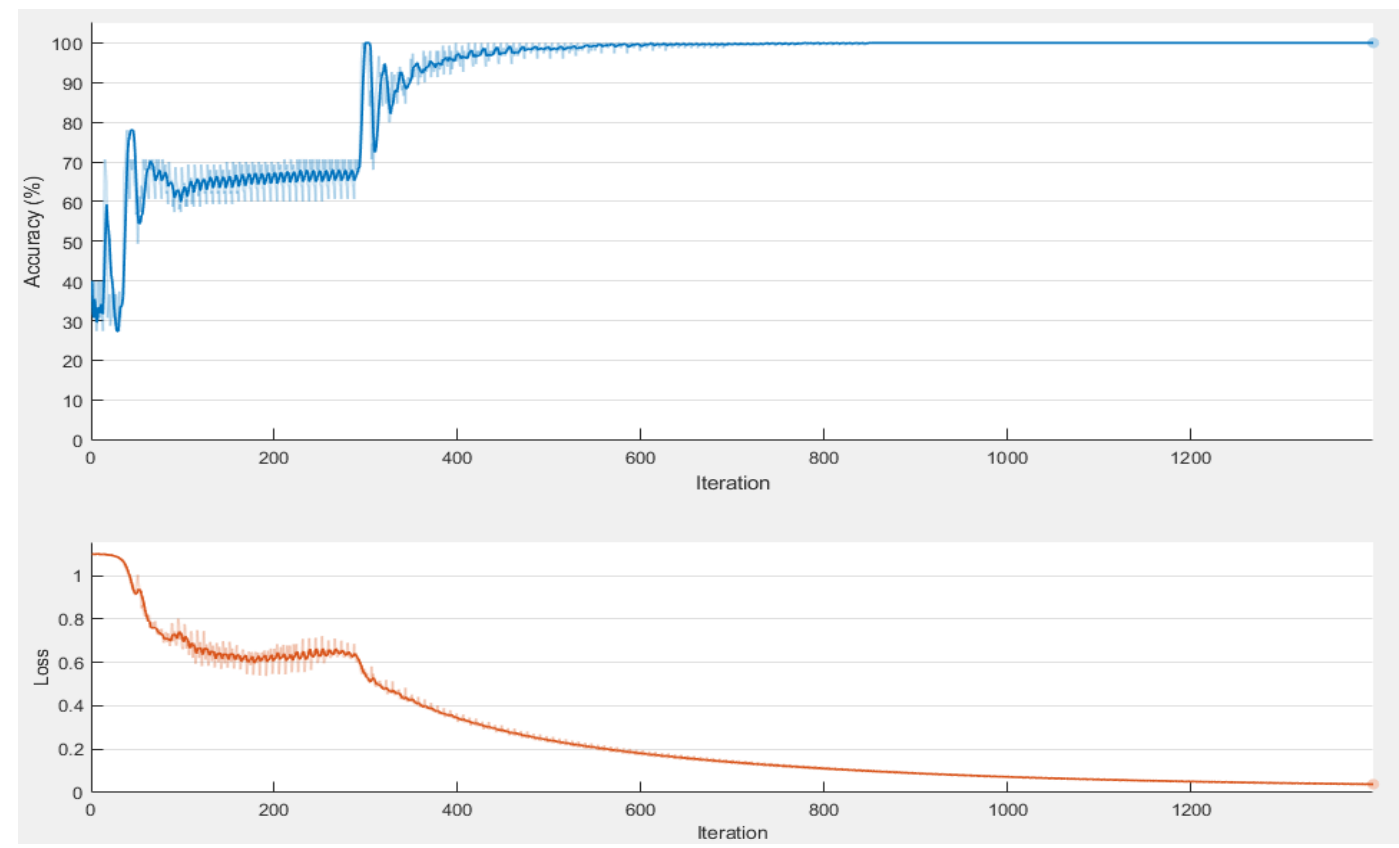

Fig. 4. Graphs of accuracy and loss in training the LSTM model.

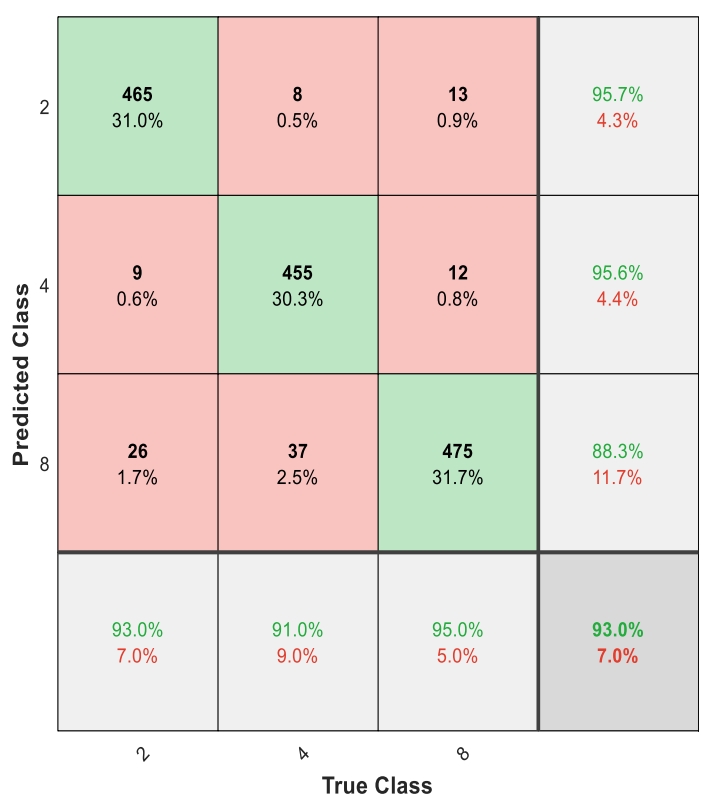

Fig. 5. Confusion matrix of classification with SNR of $0 \mathrm{~dB}$ for MPSK modulation type. Note that class labels 2,4 , and 8 denote BPSK, QPSK and 8PSK, respectively.

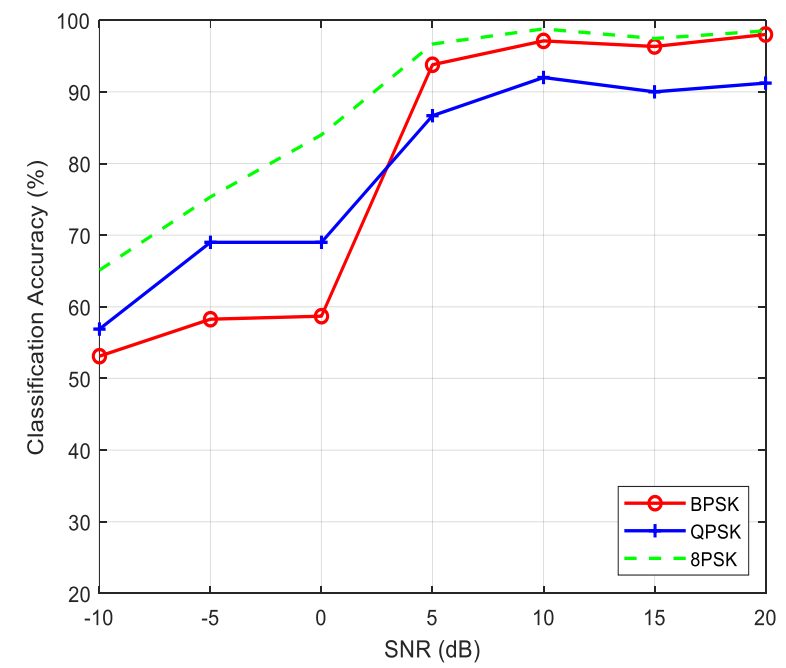

Fig. 7. Classification accuracy evaluation of the proposed LSTM model for MPSK modulation type.

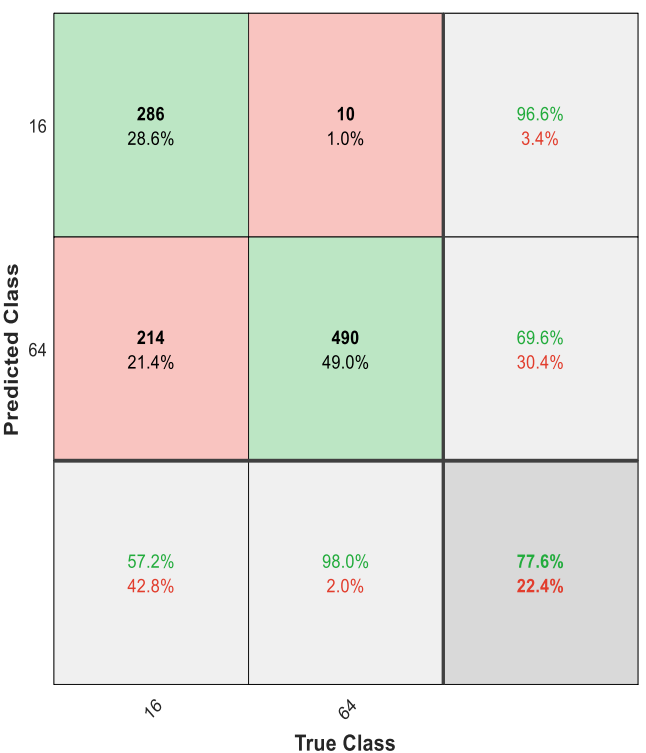

Fig. 6. Confusion matrix of classification with SNR of $0 \mathrm{~dB}$ for MQAM modulation type. Note that class labels 16 and 64 denote 16QAM and 64QAM, respectively.

In Fig. 8, higher classification accuracy is obtained by the proposed LSTM model for 64QAM compared to 16QAM. Average classification accuracy of $64.38 \%$ and $73.75 \%$ are obtained for 16QAM and 64QAM, respectively. Meanwhile, in the high SNR region (i.e. $5 \mathrm{~dB}$ and above), the model yields average classification accuracy of $93.34 \%$ for 16QAM and $97.51 \%$ for 64QAM. The reason for the performance degradation of the model in the low SNR region is due to the Rayleigh fading channel which causes severe distortion of the constellations of the modulation schemes, especially the schemes with high constellation sizes.

The results also reveal that the proposed LSTM model recognizes modulation schemes with higher constellation sizes in a group of schemes. 


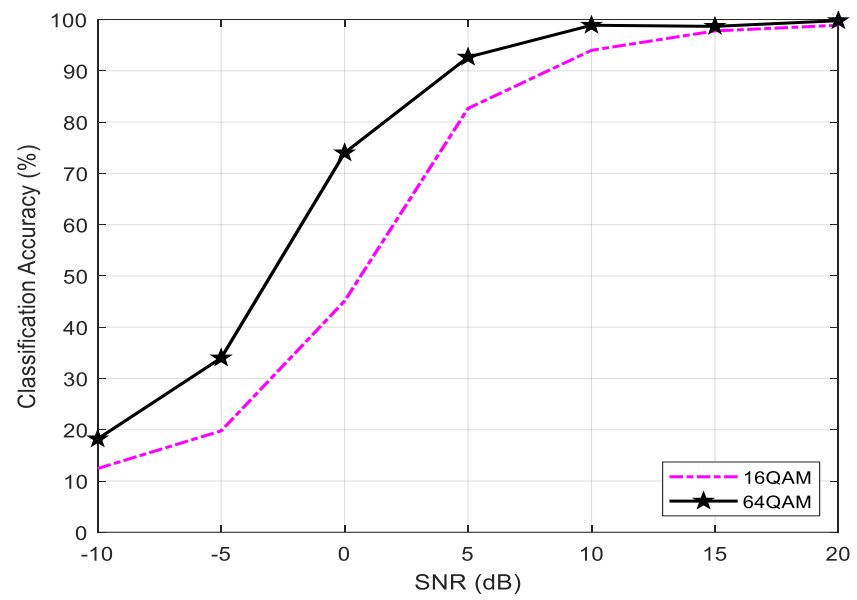

Fig. 8. Classification accuracy evaluation of the proposed LSTM model for MQAM modulation type.

\section{CONCLUSION AND FUTURE WORK}

In this paper, an LSTM model is proposed for cognitive radio networks to classify modulation schemes. The proposed LSTM model was trained for BPSK, QPSK, 8PSK, 16QAM and 64QAM digital modulation schemes with SNRs of -10 $\mathrm{dB}, 5 \mathrm{~dB}$ and $20 \mathrm{~dB}$; but can be used to classify signals of SNR from $-10 \mathrm{~dB}$ to $20 \mathrm{~dB}$. The proposed LSTM model yields an average classification accuracy of $95 \%$ with SNRs from $0 \mathrm{~dB}$ and above. This reveals the effectiveness of the proposed LSTM model in Rayleigh fading channels; and is thus suitable for practical applications in wireless communications. Future investigation of the proposed LSTM model will include analogue modulation types.

\section{REFERENCES}

[1] Mardani MR, Ghanbari M. Mohebi S. Energy and latency-aware scheduling under channel uncertainties in LTE networks for massive IoT. Wireless Personal Communications. 2018 Dec 17; 103(3):21372154.

[2] Yang K-P, McDowell P, Devkota P, Pradhan S, Bhandari R, Madewell Z. Detecting gas leaks: a case study in IoT technologies. European Journal of Engineering and Technology Research. 2021; 6(7):103-106.

[3] Salam AO. Adaptive coded modulation classification and spectrum sensing for cognitive radio systems. Ph.D. Thesis. University of Bradford; 2018.

[4] Elias FGM, Fernández EMG. An analysis of energy detector based on improved approximations of the chi-square distributions. $J$ Wireless Com Network. 2021; 44(2021): 1-18. doi: 10.1186/s13638-021-01915-5.

[5] Bahloul MR, Yusoff MZ, Abdel-Aty A-H, Saad MNM, Kudsi L. Spectrum sensing for cognitive radio systems using modulation classification: a review. Appl. Math. Inf. Sci. 2017 May 1; 11(3):857865.

[6] Al-Nuaimi DH, Hashim IA, Abidin ISZ, Salman LB, Isa NAM. Performance of feature-based techniques for automatic digital modulation recognition and classification - a review. Electronics. 2019 Nov 26; 8(1407):2-25. doi: 10.3390/electronics8121407.

[7] Kharbech S, Dayoub I, Colin MZ, Simon EP. On classifiers for blind feature-based automatic modulation classification Over Multiple-Input Multiple-Output Channels. IET Communications. 2016; 10(7):790795.

[8] Le B, Rondeau TW, Bostian CW. Modulation identification using neural networks for cognitive radios. Software Defined Radio Forum Technical Conference; 2005.

[9] Abu-Romoh M, Aboutaleb A, Rezki Z. Automatic modulation classification using moments and likelihood maximization. IEEE Communications Letters. 2018; 1(1):1-4. doi: 10.1109/lcomm.2018.2806489.

[10] Abdelbar M, Tranter B, Bose T. Cooperative modulation classification of multiple signals in cognitive radio networks. Proceedings of the
IEEE ICC 2014 - Cognitive Radio and Networks Symposium. pp. 1483 1488, Sydney, Australia, Jun 2014. doi: 10.1109/ICC.2014.6883531.

[11] Ahmed AG. Automatic modulation recognition based on artificial neural networks over wireless fading channel in cognitive radio. International Journal of Advanced Computational Engineering and Networking. 2017 Oct 17; 5(10):5-8.

[12] Sun X, Su S, Zuo Z, Guo X, Tan X. Modulation classification using compressed sensing and decision tree-support vector machine in cognitive radio system. Sensors. 2020 Mar 6; 20(1438):2-16. doi: $10.3390 / \mathrm{s} 20051438$.

[13] Salam AOA, Sheriff RE, Al-Araji SR, Mezher K, Nasir Q. Automatic modulation classification in cognitive radio using multiple antennas and maximum-likelihood techniques. Proceedings of the 2015 IEEE International Conference on Computer and Information Technology Liverpool, UK. 2015. doi: 10.1109/CIT/IUCC/DASC/PICOM.2015.3

[14] Ghauri SA, Qureshi IM, Shah I, Khan N. Modulation classification using cyclostationary features on fading channels. Research Journal of Applied Sciences, Engineering and Technology. 2014 Jun 25; 7(24):5331-5339. doi: 10.19026/rjaset.7.932.

[15] Zhang T, Shuai C, Zhou Y. Deep learning for robust automatic modulation recognition method for IoT applications. IEEE Access. 2020; 8(1):117689-117697.

[16] Zhou S, Yin Z, Wu Z, Chen Y, Zhao N, Yang Z. A robust modulation classification method using convolutional neural networks. EURASIP Journal on Advances in Signal Processing. 2019; 21(1):1-15. doi: 10.1186/s13634-019-0616-6.

[17] Ajayi OO, Badrudeen AA, Oyedeji AI. Deep learning based spectrum sensing technique for smarter cognitive radio networks. Journal of Inventive Engineering and Technology (JIET). 2021 Sep 23; 1(5):6477.

[18] Wang Y, Liu M, Yang J, Gui G. Data-driven deep learning for automatic modulation recognition in cognitive radios. IEEE Trans. Veh. Technol. 2019 Feb 20; 68(4):4074-4077.

[19] Zheng S, Qi P, Chen S, Yang X. Fusion methods for CNN-based automatic modulation classification. IEEE Access. 2019 May 22; 7(2019):66496-66504. doi 10.1109/ACCESS.2019.2918136.

[20] Kim SH, Kim JW, Nwadiugwu WP, Kim DS. Deep learning-based robust automatic modulation classification for cognitive radio networks. IEEE Access. 2021 Jul 5; 9(2021):92386-92393.

[21] Lin R, Ren W, Sun X, Yang Z, Fu K. A hybrid neural network for fast automatic modulation classification. IEEE Access. 2020 Jul 27; 8(2021):130314-130322.

[22] Zhu X. Studies on modulation classification in cognitive radios using machine learning. Ph.D. Thesis. The University of ElectroCommunications; 2017.

[23] Clement JC, Indira N, Vijayakumar P, Nandakumar R. Deep learning based modulation classification for $5 \mathrm{G}$ and beyond wireless systems. Peer-to-Peer Networking and Applications. 2021 Jan 1; 14(1): 319332. doi: 10.1007/s12083-020-01003-3.

[24] Gers FA, Schmidhuber J, Cummins F. Learning to forget: Continual prediction with LSTM. Neural Computation. 2000; 12(10):2451-2471.

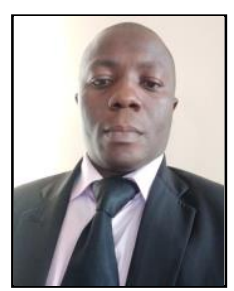

Olumide O. Ajayi received the B.Tech., M.Tech., and $\mathrm{Ph} . \mathrm{D}$ degrees in electronic and electrical engineering from the Ladoke Akintola University of Technology (LAUTECH), Ogbomoso, Oyo State, Nigeria, in 2008, 2014, and 2021, respectively.

He is currently the Acting Head in the Department of Electrical and Electronics Engineering, Adeleke University, Ede, Nigeria. His current research interests include LTE, 5G, Cognitive Radio Networks, IoT, Radio Resource Management, Deep Learning and Artificial Intelligence.

Dr. Ajayi is a member of IEEE, The Nigerian Society of Engineers

(NSE), and a Registered Engineer with the Council for the Regulation of Engineering in Nigeria (COREN). 